\title{
A radial basis Galerkin method for spherical surface Stokes equations
}

\author{
Mahadevan Ganesh ${ }^{1} \quad$ Quoc Thong Le Gia ${ }^{2}$
}

(Received 27 January 2011; revised 18 April 2011)

\begin{abstract}
Many global climate models require efficient algorithms for solving the Stokes and Navier-Stokes equations with a divergence-free constraint on a spherical surface. Compactly supported radial basis functions (with centres at well distributed mesh points on a spherical surface) are more efficient than mesh based methods for computing divergence-free numerical solutions for partial differential equations on smooth surfaces. As a stepping stone towards developing an efficient radial basis algorithm for the full Navier-Stokes equations, we propose, analyse, and implement a surface divergence-free spherical radial basis Galerkin method for the Stokes equations on the unit sphere.
\end{abstract}

\section{Contents}

1 Introduction

http://anziamj.austms.org.au/ojs/index.php/ANZIAMJ/article/view/3921 gives this article, (C) Austral. Mathematical Soc. 2011. Published May 3, 2011. IssN 1446-8735. (Print two pages per sheet of paper.) Copies of this article must not be made otherwise available on the internet; instead link directly to this URL for this article. 


\section{Preliminaries}

2.1 The Stokes equations and operators on the sphere . . . . C59

2.2 Function spaces and weak formulation . . . . . . . C60

3 A radial basis Galerkin method

3.1 Divergence-free spherical radial functions . . . . . . . . C63

3.2 Finite dimensional spaces, method and analysis . . . . . C64

4 Numerical experiments

5 Conclusions

C69

References

C70

\section{Introduction}

Simulation of the Navier-Stokes equations (NSEs) on a spherical surface, and of associated reduced forms such as the shallow water equations and the creeping flow Stokes equations, are crucial for investigation of various physical processes on the Earth's surface. One approach to advance the state of the art climate and weather prediction models is through development of efficient algorithms and associated numerical analysis for such partial differential equations (PDEs) with a divergence-free constraint on spherical surfaces.

Numerical methods for PDEs fall into two broad classes. The first class is the mesh based approach such as the finite-element method (FEM). The mesh based methods for approximating a smooth solution of a PDE on a smooth geometry, in general, lead to low order $L^{2}$-norm accuracy $\mathcal{O}\left(h^{r}\right)$ (typically $r=2$ ). Here, $h$ is the maximum diameter of elements in the triangulation of the geometry and $r$ is the order of spline basis functions. However, the Galerkin FEM is an efficient numerical method for PDEs on complicated non-smooth geometries. For a fixed $h$ and $r$, each spline basis function is locally supported on the triangulation. Hence the Galerkin FEM 
requires setting up and solving a sparse linear system with each entry being a Galerkin integral only on a small patch of the triangulation.

The other class of numerical methods for PDEs is the meshless approach such as the spectral and radial basis methods. A fully discrete spectral Galerkin method, that does not require mesh points and an associated triangulation of the sphere, was recently investigated by us for the NSEs on a spherical surface [2]. Ganesh et al. [2] proved and demonstrated that the meshless spectral method is a high order method. However, the polynomial basis functions in the spectral method for the NSES are globally supported on the sphere, leading to dense algebraic systems.

Here we develop a Galerkin method for the Stokes equations on the unit sphere using surface-divergence-free, compactly supported radial basis functions. This meshless approach requires construction of well distributed mesh points on the sphere (so that the mesh norm $h$ is small) and associated tangent vectors. The algorithm has advantages of both the Galerkin FEM and spectral methods. The vector-valued basis functions with high order $(\tau)$ smoothness corresponding to the points can be set up easily without any triangulation of the sphere. For a fixed $h$ and $\tau$, the meshless algorithm for the Stokes equations requires setting up and solving a symmetric positive definite sparse linear system. The entries of the linear system are Galerkin integrals defined only on the supports of the radial basis functions. We prove and numerically demonstrate that the radial basis Galerkin method, for smooth solutions, is of high order with accuracy $\mathcal{O}\left(h^{2 \tau-1}\right)$. Further, we show that the method is also useful for approximating non-smooth solutions of the Stokes equations, with an order of accuracy restricted by low regularity of the solution.

The next section describes the Stokes equations and associated operators and divergence-free function spaces on the sphere. Section 3 gives the surfacedivergence-free radial basis Galerkin method and its numerical analysis. Numerical experiments given in Section 4 demonstrate the theory and applicability of the method to simulate some benchmark smooth and non-smooth Stokes flows on the sphere. 


\section{Preliminaries}

\subsection{The Stokes equations and operators on the sphere}

The Stokes equations on the unit sphere $S \subset \mathbb{R}^{3}$ are written as [3]

$$
-v \Delta \mathbf{u}+\frac{1}{\rho} \operatorname{Grad} p=\mathbf{F}, \quad \operatorname{Div} \mathbf{u}=0, \quad \text { on } S,
$$

where $\mathbf{u}=\left(\mathfrak{u}_{1}(\mathbf{x}), \mathfrak{u}_{2}(\mathbf{x}), \mathfrak{u}_{3}(\mathbf{x})\right)$ is the unknown tangential velocity field of the fluid at $\mathbf{x} \in S$, and the positive constants $v$ and $\rho$ are respectively the viscosity and density, $\mathbf{p}(\mathbf{x})$ is the unknown pressure field, and $\mathbf{F}$ is the external force.

In the PDEs (1), the scalar valued Div and vector valued Grad and $-\Delta$ are respectively the surface divergence, surface gradient, and the Laplace-de Rham operators. The Laplace-de Rham operator $-\Delta=\mathrm{Curl} \mathrm{Curl}_{\mathrm{x}}-\mathrm{Grad}_{\mathrm{Div}}$ restricted to the tangential divergence-free fields on the sphere is the Stokes operator $A=$ Curl Curl $_{\mathbf{x}}$.

These surface differential operators can be defined using appropriate projections of the corresponding operators in $\mathbb{R}^{3}[3,5]$. Any point $\mathbf{x} \in \mathbf{S}$, viewed as a vector in $\mathbb{R}^{3}$, equals the outward unit normal $\mathbf{n}(\mathbf{x})$ to $S$ at $\mathbf{x}$. The spherical surface gradient of a scalar function $v$ defined on $S$ is

$$
\operatorname{Grad} v=\mathrm{P}_{\mathbf{x}} \nabla v
$$

where $\mathrm{P}_{\mathbf{x}}:=\mathrm{I}-\mathbf{x} \mathbf{x}^{\top}$ projects vectors in $\mathbb{R}^{3}$ onto the tangent plane $\mathrm{TS}_{\mathbf{x}}$ (matrix I denotes the $3 \times 3$ identity matrix). The tangential spherical surface operator Curl $=-\mathbf{x} \times$ Grad, defined for a scalar function $v$ or a normal vector field $\mathbf{w}=\mathbf{x} \boldsymbol{v}$ on $S$, is written, using the vector valued operator $Q_{\mathbf{x}} \mathbf{y}:=-\mathbf{x} \times \mathbf{y}$ as

$$
\text { Curl } \mathbf{w}:=\operatorname{Curl} v=\mathrm{Q}_{\mathrm{x}} \nabla v, \quad \mathrm{Q}_{\mathrm{x}}=\left[\begin{array}{ccc}
0 & x_{3} & -x_{2} \\
-x_{3} & 0 & x_{1} \\
x_{2} & -x_{1} & 0
\end{array}\right]
$$


For a tangential vector field $\mathbf{v}$ on $\mathbf{S}$, we define the normal vector field $\operatorname{Curl}_{\mathbf{x}} \mathbf{v}$ as the projection of vector $\nabla \times \mathbf{v}$ (the usual curl in $\mathbb{R}^{3}$ ) to the unit normal vector of the surface of $S$ :

$$
\operatorname{Curl}_{\mathbf{x}} \mathbf{v}=\mathbf{x}[(\nabla \times \mathbf{v}) \cdot \mathbf{x}] .
$$

We sometimes use the standard parametrisation of a point $\mathbf{x}$ on $S$,

$$
\mathbf{x}=\mathbf{p}(\theta, \phi)=(\sin \theta \cos \phi, \sin \theta \sin \phi, \cos \theta) .
$$

and the associated orthonormal basis for the tangent space TS,

$$
\begin{aligned}
& \mathbf{e}(\theta, \phi)=(\cos \theta \cos \phi, \cos \theta \sin \phi,-\sin \theta) \\
& \mathbf{f}(\theta, \phi)=(-\sin \phi, \cos \phi, 0) .
\end{aligned}
$$

\subsection{Function spaces and weak formulation}

We recall some details that are required for describing and analysing a radial basis Galerkin method for PDEs (1) [2, 3, e.g.]. The standard inner products on the space of all square integrable scalar functions on $S$, denoted by $\mathrm{L}^{2}(\mathrm{~S})$, and tangential vector fields on $S$, denoted by $\mathrm{L}^{2}(\mathrm{TS})$, are respectively

$$
\begin{aligned}
& \left\langle v_{1}, v_{2}\right\rangle=\left\langle v_{1}, v_{2}\right\rangle_{\mathrm{L}^{2}(\mathrm{~S})}=\int_{\mathrm{S}} v_{1} \overline{v_{2}} \mathrm{~d} S, \quad v_{\mathrm{j}} \in \mathrm{L}^{2}(\mathrm{~S}), \\
& \left\langle\mathbf{v}_{1}, \mathbf{v}_{2}\right\rangle=\left\langle\mathbf{v}_{1}, \mathbf{v}_{2}\right\rangle_{\mathrm{L}^{2}(\mathrm{TS})}=\int_{\mathrm{S}} \mathbf{v}_{1} \cdot \overline{\mathbf{v}_{2}} \mathrm{~d} S, \quad \mathbf{v}_{\mathbf{j}} \in \mathrm{L}^{2}(\mathrm{TS}) .
\end{aligned}
$$

Throughout this article, the induced norm on $\mathrm{L}^{2}(\mathrm{TS})$ is denoted by $\|\cdot\|$ and for other inner product spaces, say $X$ with inner product $\langle\cdot, \cdot\rangle_{X}$, the associated norm is denoted by $\|\cdot\|_{X}$. For example, for $\mu>0$, norms in the scalar and vector valued function Sobolev spaces $\mathrm{H}^{\mu}(S)$ and $\mathrm{H}^{\mu}(\mathrm{TS})$ are denoted by $\|\cdot\|_{H^{\mu}(S)}$ and $\|\cdot\|_{H^{\mu}(T S)}$, respectively. In particular,

$$
\|\mathbf{v}\|_{H^{\mu}(\mathrm{TS})}^{2}=\|\mathbf{v}\|^{2}+\left\|(-\Delta)^{\mu / 2} \mathbf{v}\right\|^{2}
$$


Throughout, we identify a normal vector field $\mathbf{w}$ with a scalar field, and hence

$$
\left\langle\mathbf{w}_{1}, \mathbf{w}_{2}\right\rangle:=\left\langle w_{1}, w_{2}\right\rangle_{\mathrm{L}^{2}(S)}, \quad \mathbf{w}_{1}=\mathbf{x} w_{1}, \quad \mathbf{w}_{2}=\mathbf{x} w_{2}, \quad w_{1}, w_{2} \in \mathrm{L}^{2}(S) .
$$

The following identities hold for appropriate scalar and vector fields [3]

$$
\langle\operatorname{Grad} v, \mathbf{v}\rangle=-\langle v, \operatorname{Div} \mathbf{v}\rangle, \quad\left\langle\operatorname{Curl~Curl}_{\mathbf{x}} \mathbf{w}, \mathbf{z}\right\rangle=\left\langle\operatorname{Curl}_{\mathbf{x}} \mathbf{w}, \operatorname{Curl}_{\mathbf{x}} \mathbf{z}\right\rangle .
$$

In the first identity of (9), the $\mathrm{L}^{2}$ (TS) inner product is used on the left hand side and the $\mathrm{L}^{2}(\mathrm{~S})$ inner product is used on the right hand side.

Using that Div Curl $v=0$ for any scalar field $v$ on $S$, smooth $\left(\mathrm{C}^{\infty}\right)$ tangential fields on $S$ are decomposed into two components, one in the space of all divergence-free fields and the other, its orthogonal complement, through the Hodge decomposition theorem

$$
\mathrm{C}^{\infty}(\mathrm{TS})=\left\{\operatorname{Grad} \psi: \psi \in \mathrm{C}^{\infty}(\mathrm{S})\right\} \oplus \mathrm{C}^{\infty}(\mathrm{TS} ; \mathrm{Curl}),
$$

where

$$
\mathrm{C}^{\infty}(\mathrm{TS} ; \mathrm{Curl})=\left\{\operatorname{Curl} \psi: \psi \in \mathrm{C}^{\infty}(\mathrm{S})\right\} .
$$

For $\mu \geqslant 0$, let $C^{\infty, \mu}\left(\mathrm{TS}\right.$; Curl) denote the closure of $\mathrm{C}^{\infty}$ (TS; Curl) in the $\mathrm{H}^{\mu}(\mathrm{TS})$ norm. In particular, the space [3]

$$
\mathrm{V}=\text { closure of } \mathrm{C}^{\infty}(\mathrm{TS} ; \mathrm{Curl}) \text { in } \mathrm{H}^{1}(\mathrm{TS})
$$

is crucial for uncoupling the unknown velocity and pressure terms in the Stokes PDEs (1). Using (7) and (9), we obtain

$$
\|\mathbf{v}\|_{\mathrm{H}^{1}(\mathrm{TS})}^{2}=\|\mathbf{v}\|^{2}+\left\|\operatorname{Curl}_{\mathbf{x}} \mathbf{v}\right\|_{\mathrm{L}^{2}(\mathrm{~S})}^{2}, \quad \mathbf{v} \in \mathbf{V} .
$$

For $\mathbf{v}=\operatorname{Curl} \psi$, expanding $\psi$ using the $\mathrm{L}^{2}(\mathrm{~S})$ orthonormal spherical harmonics basis $Y_{l, j}$, of degree $l$ and index $\boldsymbol{j}, \boldsymbol{l}=0,1,2, \ldots,|j| \leqslant l$, using (9) and the spectral property of $\operatorname{Curl~Curl}_{\mathbf{x}}=-\Delta$ on $\mathrm{V}$, we obtain

$$
\mathbf{v}=\sum_{\mathrm{l}=1}^{\infty} \sum_{\mathrm{j} j \leqslant l}\left\langle\psi, \mathrm{Y}_{\mathrm{l}, j}\right\rangle_{\mathrm{L}^{2}(\mathrm{~S})} \operatorname{Curl}_{\mathrm{l}, \mathrm{j}}, \quad\left\|\operatorname{Curl}_{\mathbf{x}} \mathbf{v}\right\|_{\mathrm{L}^{2}(S)}^{2}=\left\langle\operatorname{Curl~Curl}_{\mathbf{x}} \mathbf{v}, \mathbf{v}\right\rangle
$$


and

$$
\operatorname{Curl~Curl}_{\mathbf{x}} \mathbf{v}=\sum_{l=1}^{\infty} \sum_{|j| \leqslant l} l(l+1)\left\langle\psi, Y_{l, j}\right\rangle_{L^{2}(S)} \operatorname{Curl}_{l, j} .
$$

The Poincaré inequality $\|\mathbf{v}\|^{2} \leqslant \frac{1}{2}\left\|\operatorname{Curl}_{\mathbf{x}} \mathbf{v}\right\|_{\mathrm{L}^{2}(\mathrm{~S})}^{2}$ follows for $\mathbf{v} \in \mathrm{V}$. Consequently, using (13), the $\mathrm{H}^{1}$ (TS) norm is equivalent to the energy norm $\|\mathbf{v}\|_{\mathrm{V}}^{2}=\langle A \mathbf{v}, \mathbf{v}\rangle=\left\|\operatorname{Curl}_{\mathbf{x}} \mathbf{v}\right\|^{2}$ on $\mathbf{V}$

$$
\left\|\operatorname{Curl}_{\mathbf{x}} \mathbf{v}\right\|_{\mathrm{L}^{2}(S)}^{2} \leqslant\|\mathbf{v}\|_{\mathrm{H}^{1}(\mathrm{TS})}^{2} \leqslant \frac{3}{2}\left\|\operatorname{Curl}_{\mathbf{x}} \mathbf{v}\right\|_{\mathrm{L}^{2}(S)}^{2}, \quad \mathbf{v} \in \mathbf{V}
$$

Using (1), (9), (14), and that $v>0$, we obtain the following weak formulation of the spherical surface Stokes equations

$$
v\left\langle\operatorname{Curl}_{\mathbf{x}} \mathbf{u}, \operatorname{Curl}_{\mathbf{x}} \mathbf{v}\right\rangle=\langle\mathbf{F}, \mathbf{v}\rangle, \quad \mathbf{v} \in \mathrm{V} .
$$

The left hand side of (15) defines a bounded, symmetric, and coercive bilinear form on $\mathrm{V}$, so the existence and uniqueness of the solution $\mathbf{u} \in \mathrm{V}$ of (15) follows from the Lax-Milgram theorem, for all $\mathbf{F}$ in $\mathrm{V}^{*}$, the adjoint of $\mathrm{V}$.

Applying the Div operator on both sides of (1), we obtain

$$
\frac{1}{\rho} \Delta \mathrm{p}=\operatorname{Div} \mathrm{F} \quad \text { on } \mathrm{S} \text {. }
$$

Hence the pressure term, if required, is computed using established mesh based or meshless Poisson solvers. We focus on developing, analysing, and implementing a radial basis Galerkin method for (15).

\section{A radial basis Galerkin method}

For a smoothness parameter $\tau$, we first construct finite dimensional subspaces of $\mathrm{V} \cap \mathrm{H}^{\tau}(\mathrm{TS})$ spanned by tangential, surface-divergence-free, compactly supported spherical radial basis functions and conclude this section by proving that, if (15) has a solution $\mathbf{u} \in \mathrm{V} \cap \mathrm{H}^{2 \tau-1}(\mathrm{TS})$, the radial basis Galerkin solutions converge to $\mathbf{u}$ with rate of convergence $2 \tau-1$ in the $\mathrm{L}^{2}$-norm. 


\subsection{Divergence-free spherical radial functions}

We motivate the construction of a vector valued Galerkin method by starting with a class of scalar valued compactly supported radial functions $\psi_{\tau}$ defined on $[0, \infty)$, introduced by Wendland [7]. Throughout this section, the smoothness parameter is $\tau=m / 2$ for some odd integer $m \geqslant 3$.

For the numerical experiments, $m=7$ or 9 , and we use

$$
\begin{aligned}
& \psi_{7 / 2}(r)=\left(1+8 r+25 r^{2}+32 r^{3}\right)(1-r)_{+}^{8}, \\
& \psi_{9 / 2}(r)=\left(1+10 r+42 r^{2}+90 r^{3}+429 r^{4} / 5\right)(1-r)_{+}^{10},
\end{aligned}
$$

as scalar radial basis functions. We demonstrate high order rates of convergence for a benchmark smooth Stokes flow. However, for a non-smooth Stokes flow in $\mathrm{V} \cap \mathrm{H}^{\beta}$ (TS) with $2<\beta<3$, as proved in this section and demonstrated using a benchmark example in Section 4 , the rate of convergence is at most $\beta$ despite using $\psi_{7 / 2}$ and $\psi_{9 / 2}$.

For $\mathbf{x}, \mathbf{y} \in \mathbf{S}$, motivated by the observation that $\|\mathbf{x}-\mathbf{y}\|=\sqrt{2-2 \mathbf{x} \cdot \mathbf{y}}$, we define

$$
\phi_{\tau}(t)=\psi_{\tau}(\sqrt{2-2 t}), \quad t \in[-1,1] .
$$

For all $\ell \geqslant 0$, let $P_{\ell}$ denote the Legendre polynomial of degree $\ell$. The $\ell$ th Legendre-Fourier coefficient, $\widehat{\phi_{\tau, \ell}}=2 \pi \int_{-1}^{1} \phi_{\tau}(t) P_{\ell}(t) d t$, of $\phi_{\tau}$ is positive and decays like $(\ell+1)^{-2(\tau+1)}$ as $\ell \rightarrow \infty$ [4]. That is, there exist positive constants $\mathrm{c}_{1}$ and $\mathrm{c}_{2}$ such that

$$
c_{1}(\ell+1)^{-2(\tau+1)} \leqslant \widehat{\phi_{\tau, \ell}} \leqslant c_{2}(\ell+1)^{-2(\tau+1)}, \quad \ell \geqslant 0 .
$$

The convergence analysis here requires only properties (19)-(20). Hence the divergence-free radial basis Galerkin method and analysis are applicable for any class of scalar radial basis functions $\psi_{\tau}$ satisfying (19)-(20). The Wendland scalar radial basis functions are widely used due to various properties and robust theoretical results [7] and hence we choose these functions for motivation and in our numerical experiments. 
Since Div Curl $v=0$ for any scalar field, we obtain tangential surfacedivergence-free radial basis functions (with various centres $\mathbf{y}$ ) by applying the Curl $=\mathrm{Q}_{\mathbf{x}} \nabla$ operator to $\psi_{\tau}(\|\mathbf{x}-\mathbf{y}\|)$. However, in this case the crucial positive definiteness of the associated kernel need not hold. This problem is avoided by applying the operator $\mathrm{Curl} \mathrm{Curl}^{\top}$. That is, as suggested by Narcowich et al. $[1,5]$, we consider the matrix valued radial basis kernel

$$
\Psi_{\tau}(\mathbf{x}, \mathbf{y})=\mathrm{Q}_{\mathbf{x}}\left(-\nabla \nabla^{\top} \psi_{\tau}(\|\mathbf{x}-\mathbf{y}\|)\right) \mathrm{Q}_{\mathbf{y}}^{\top} .
$$

Here $\nabla \nabla^{\top} \psi_{\tau}(\|\mathbf{x}\|)=\left[\partial_{x_{j}} \partial_{x_{k}} \psi_{\tau}(\|\mathbf{x}\|)\right]$ is the $3 \times 3$ Hessian matrix. Since Div Curl $=0$,

$$
\operatorname{Div}\left(\Psi_{\tau}(\cdot, \mathbf{y}) \mathbf{t}_{\mathbf{y}}\right)=-\operatorname{Div} \operatorname{Curl}\left(\nabla^{\top} \Psi_{\tau}(\|\cdot-\mathbf{y}\|) \mathrm{Q}_{\mathbf{y}}^{\top} \mathbf{t}_{\mathbf{y}}\right)=0, \quad \mathbf{t}_{\mathbf{y}} \in \mathrm{TS}_{\mathbf{y}},
$$

and hence, using $(20), \Psi_{\tau}(\cdot, \mathbf{y}) \mathbf{t}_{\mathbf{y}} \in \mathrm{V} \cap \mathrm{H}^{\tau}\left(\mathrm{TS}_{\mathbf{y}}\right)$ for all $\mathbf{t}_{\mathbf{y}} \in \mathrm{TS}_{\mathbf{y}}[1]$.

\subsection{Finite dimensional spaces, method and analysis}

Denote by $\theta(\mathbf{x}, \mathbf{y})=\cos ^{-1}(\mathbf{x} \cdot \mathbf{y})$ the geodesic distance between two points $\mathbf{x}$ and $\mathbf{y}$ on the sphere. Suppose $Y_{N}=\left\{\mathbf{y}_{1}, \ldots, \mathbf{y}_{N}\right\} \subset S$ is a set of well distributed points on $S$, so that the density of the set, measured through the mesh norm

$$
h_{N}=\sup _{\mathbf{x} \in S} \min _{1 \leqslant i \leqslant N} \theta\left(\mathbf{x}, \mathbf{y}_{i}\right),
$$

satisfies $0<h_{N}<1$. Another quantity of interest from the set $Y_{N}$, to analyse the approximation of non-smooth functions [1], is the separation radius

$$
\mathrm{q}_{\mathrm{N}}=\frac{1}{2} \min _{1 \leqslant i \neq j \leqslant N} \theta\left(\mathbf{y}_{j}, \mathbf{y}_{\mathrm{i}}\right)
$$

Similar to a quasi-uniform mesh requirement in the FEM, for optimal approximation of non-smooth functions with respect to $h_{N}$, it is important to have well distributed points on the sphere so that $q_{N}=\mathcal{O}\left(h_{N}\right)$. There are several 
approaches to construct the set $Y_{N}$ and, for our numerical experiments we chose a simple method based on an analytical formula [6].

At each point $\mathbf{y}_{j}=\mathbf{p}\left(\theta_{j}, \phi_{j}\right) \in Y_{N}(j=1, \ldots, N)$ we define an orthonormal basis for the tangential space $\mathrm{TS}_{\mathbf{y}_{j}}$ consisting of the vectors $\mathbf{e}_{j}=\mathbf{e}\left(\theta_{j}, \phi_{j}\right)$ and $\mathbf{f}_{j}=\mathbf{f}\left(\theta_{j}, \phi_{j}\right)$. Using $(21)$ on the set of points in $Y_{N}$ we define $\Psi_{\tau, j}:=\Psi_{\tau}\left(\cdot, \mathbf{y}_{j}\right)$, and thereby obtain $2 \mathrm{~N}$ linearly independent, vector valued, tangential, surfacedivergence-free radial basis functions in $\mathrm{V} \cap \mathrm{H}^{\tau}(\mathrm{TS})$,

$$
\Psi_{\tau, j} \mathbf{e}_{j}, \quad \Psi_{\tau, j} \mathbf{f}_{j}, \quad j=1, \ldots, N .
$$

The $2 \mathrm{~N}$-dimensional subspace of $\mathrm{V} \cap \mathrm{H}^{\tau}(\mathrm{TS})$ in which we seek an approximate solution of the Stokes equation is

$$
V_{\tau, h_{N}}=\operatorname{span}\left\{\Psi_{\tau, j} \mathbf{e}_{j}, \Psi_{\tau, j} \mathbf{f}_{j}: j=1, \ldots, N\right\} .
$$

The Galerkin method for the spherical surface Stokes equation (1) is to find $\mathbf{u}_{\tau, h_{N}} \in V_{\tau, h_{N}}$ such that

$$
v\left\langle\operatorname{Curl}_{\mathbf{x}} \mathbf{u}_{\tau, h_{N}}, \operatorname{Curl}_{\mathbf{x}} \mathbf{v}\right\rangle=\langle\mathbf{F}, \mathbf{v}\rangle, \quad \mathbf{v} \in \mathrm{V}_{\tau, h_{N}} .
$$

We represent the unknown unique vector field $\mathbf{u}_{\tau, h_{N}} \in V_{\tau, h_{N}}$ satisfying (25) as

$$
\mathbf{u}_{\tau, h_{N}}=\sum_{i=1}^{N}\left[\alpha_{i} \Psi_{\tau, i} \mathbf{e}_{i}+\beta_{i} \Psi_{\tau, i} \mathbf{f}_{i}\right]
$$

Taking $\mathbf{v}=\Psi_{j} \mathbf{e}_{j}$ in (25), the $2 \mathrm{~N}$ unknowns in (26) are computed by solving the sparse, symmetric, and positive-definite system

$$
\sum_{i=1}^{N} M_{j, i}\left[\begin{array}{l}
\alpha_{i} \\
\beta_{i}
\end{array}\right]=\left[\begin{array}{l}
\left\langle\mathbf{F}, \Psi_{j} \mathbf{e}_{j}\right\rangle \\
\left\langle\mathbf{F}, \Psi_{j} \mathbf{f}_{j}\right\rangle
\end{array}\right], \quad j=1, \ldots, N,
$$

where the $2 \times 2$ symmetric blocks $M_{j, i}$ for $i, j=1, \ldots, N$ are

$$
M_{j, i}=\left[\begin{array}{ll}
\left\langle\operatorname{Curl}_{\mathbf{x}} \Psi_{\tau, i} \mathbf{e}_{i}, \operatorname{Curl}_{\mathbf{x}} \Psi_{\tau, j} \mathbf{e}_{\mathbf{j}}\right\rangle & \left\langle\operatorname{Curl}_{\mathbf{x}} \Psi_{\tau, \mathbf{f}} \mathbf{f}_{\mathbf{i}}, \operatorname{Curl}_{\mathbf{x}} \Psi_{\tau, j} \mathbf{e}_{\mathbf{i}}\right\rangle \\
\left\langle\operatorname{Curl}_{\mathbf{x}} \Psi_{\tau, \mathbf{i}} \mathbf{e}_{\mathbf{i}}, \operatorname{Curl}_{\mathbf{x}} \Psi_{\tau, j} \mathbf{f}_{\mathbf{j}}\right\rangle & \left\langle\operatorname{Curl}_{\mathbf{x}} \Psi_{\tau, \mathbf{i}} \mathbf{f}_{\mathbf{i}}, \operatorname{Curl}_{\mathbf{x}} \Psi_{\tau, j} \mathbf{f}_{\mathbf{j}}\right\rangle
\end{array}\right]
$$


Using standard duality arguments, we obtain the convergence and superconvergence of the Galerkin approximations. Throughout the remainder of this article, $C$ is a generic constant, independent of $N, h_{N}$ and $q_{N}$.

Theorem 1 Let $\mathbf{u} \in \mathrm{V}$ and $\mathbf{u}_{\tau, h_{\mathrm{N}}} \in \mathrm{V}_{\tau, h_{\mathrm{N}}}$ be the unique solutions of (15) and (25), respectively. Then for $\mu \in[0,1]$ and $1<\beta \leqslant \tau$,

$$
\left\|\mathbf{u}-\mathbf{u}_{\tau, h_{N}}\right\|_{H^{\mu}(T S)} \leqslant \begin{cases}C_{N}^{\tau-\mu} q_{N}^{\beta-\tau}\|\mathbf{u}\|_{H^{\beta}(T S)}, & \text { if } \mathbf{u} \in \mathrm{V} \cap \mathrm{H}^{\beta}(\mathrm{TS}), \\ \mathrm{Ch}_{\mathrm{N}}^{(2 \tau-\mu-1)}\|\mathbf{u}\|_{H^{2 \tau-1}(\mathrm{TS})}, & \text { if } \mathbf{u} \in \mathrm{V} \cap \mathrm{H}^{2 \tau-1}(\mathrm{TS}) .\end{cases}
$$

Proof: Using (15) and (25),

$$
\left\langle\operatorname{Curl}_{\mathbf{x}}\left(\mathbf{u}-\mathbf{u}_{\tau, h_{N}}\right), \operatorname{Curl}_{\mathbf{x}} \mathbf{v}\right\rangle=0, \quad \mathbf{v} \in \mathrm{V}_{\tau, h_{N}} .
$$

If $\mathbf{v} \in \mathbf{V}$, there exists a unique interpolant $\mathcal{J}_{\tau, h_{N}} \mathbf{v} \in \mathrm{V}_{\tau, h_{N}}$ with $\mathcal{J}_{\tau, h_{N}} \mathbf{v}=\mathbf{v}$ on $Y_{N}$ such that (27) holds with $\mathbf{u}$ and $\mathbf{u}_{\tau, h_{N}}$ respectively replaced with $\mathbf{v}$ and $\mathcal{J}_{\tau, h_{N}} \mathbf{v}$ [1]. Hence, using (14) and (28), the inequalities in (27) for $\mu=1$ follow from the bound

$$
\begin{aligned}
\left\|\mathbf{u}-\mathbf{u}_{\tau, h_{N}}\right\|_{\mathrm{H}^{1}(\mathrm{TS})}^{2} & \leqslant \frac{3}{2}\left\langle\operatorname{Curl}_{\mathbf{x}}\left(\mathbf{u}-\mathbf{u}_{\tau, h_{N}}\right), \operatorname{Curl}_{\mathbf{x}}\left(\mathbf{u}-\mathcal{J}_{\tau, h_{N}} \mathbf{u}\right)\right\rangle \\
& \leqslant \frac{3}{2}\left\|\mathbf{u}-\mathbf{u}_{\tau, h_{N}}\right\|_{\mathrm{H}^{1}(\mathrm{TS})}\left\|\mathbf{u}-\mathcal{J}_{\tau, h_{N}} \mathbf{u}\right\|_{\mathrm{H}^{1}(\mathrm{TS})} .
\end{aligned}
$$

Let $\mathbf{w} \in \mathrm{V} \cap \mathrm{H}^{2}(\mathrm{TS})$ be the unique solution of $-\Delta \mathbf{w}=\mathbf{u}-\mathbf{u}_{\tau, h_{\mathrm{N}}}$. Using the approximation power of $\mathcal{J}_{\tau, h_{\mathbb{N}}} \mathbf{W}$ and (7), we obtain

$$
\left\|\mathbf{w}-\mathcal{J}_{\tau, h_{N}} \mathbf{w}\right\|_{H^{1}(T S)} \leqslant C h_{N}\|\mathbf{w}\|_{H^{2}(T S)} \leqslant C h_{N}\left\|\mathbf{u}-\mathbf{u}_{\tau, h_{N}}\right\| .
$$

Since

$$
\left\langle\operatorname{Curl}_{\mathbf{x}} \mathbf{w}, \operatorname{Curl}_{\mathbf{x}} \mathbf{v}\right\rangle=\langle-\Delta \mathbf{w}, \mathbf{v}\rangle=\left\langle\mathbf{u}-\mathbf{u}_{\tau, h_{\mathrm{N}}}, \mathbf{v}\right\rangle, \quad \mathbf{v} \in \mathbf{V},
$$

using (28),

$$
\left\|\mathbf{u}-\mathbf{u}_{\tau, h_{N}}\right\|^{2}=\left\langle\operatorname{Curl}_{\mathbf{x}}\left(\mathbf{w}-\mathcal{J}_{\tau, h_{N}} \mathbf{w}\right), \operatorname{Curl}_{\mathbf{x}}\left(\mathbf{u}-\mathbf{u}_{\tau, h_{N}}\right)\right\rangle
$$




$$
\leqslant\left\|\mathbf{w}-\mathcal{J}_{\tau, h_{N}} \mathbf{W}\right\|_{H^{1}(T S)}\left\|\mathbf{u}-\mathbf{u}_{\tau, h_{N}}\right\|_{H^{1}(T S)} .
$$

Now the results in (27) for $\mu=0$ follow from (31), (29) and (27) with $\mu=1$.

For the non-smooth solution, $\mathbf{u} \in \mathrm{V} \cap \mathrm{H}^{\beta}(\mathrm{TS}), 1<\beta \leqslant \tau$, if we assume the quasi-uniform condition, $\mathbf{q}_{\mathrm{N}} \leqslant \mathrm{Ch}_{\mathrm{N}}$, using the first inequality in (27), we obtain the optimal order convergence $\left\|\mathbf{u}-\mathbf{u}_{\tau, h_{N}}\right\|_{H^{\mu}(\mathrm{TS})}=\mathcal{O}\left(h_{N}^{\beta-\mu}\right)$.

\section{Numerical experiments}

We demonstrate the tangential surface-divergence-free radial basis Galerkin method and analysis using two test vector fields. In our first example, $\mathbf{u} \in \mathrm{V} \cap \mathrm{H}^{\beta}$ (TS) is a smooth field with $\beta>2 \tau$, and for the second nonsmooth case, $2<\beta<3$ with $\beta<\tau$. A standard approach to generate such benchmark vector fields in the atmospheric sciences, to demonstrate validity of numerical algorithms on the sphere, is through various stream functions [8]. For any scalar stream function $u \in H^{\beta+1}(S)$ it is easy to verify that the vector field

$$
\mathbf{u}(\mathbf{x})=\frac{1}{\sin \theta} \frac{\partial \mathbf{u}}{\partial \phi} \mathbf{e}-\frac{\partial \mathbf{u}}{\partial \theta} \mathbf{f}
$$

satisfies $\mathbf{u} \in \mathrm{V} \cap \mathrm{H}^{\beta}$ (TS). In our numerical experiments, the forcing field $\mathbf{F}$ is chosen so that $\mathbf{u}$ in (32) is the unique solution of the weak form of the Stokes equation (15) with $v=1$. All our experiments were performed in Matlab on a desktop machine equipped with Intel Core 2 Duo $3.1 \mathrm{GHz}$ processor and $4 \mathrm{~GB}$ of RAM.

Example 1 Motivated by the benchmark atmospheric low pressure flow field (popularly known as the Test Case $4[5,8]$ ) centred at $\mathbf{x}_{\mathbf{c}}=\mathbf{p}(\pi / 4,0)$, 
for the first smooth test field, we choose $\boldsymbol{u}$ in (32) so that

$$
u(x)=\frac{2}{3} \sin ^{15} \theta-u_{1, \mathbf{x}_{c}, \sigma_{1}}(x), \quad u_{1, \mathbf{x}_{\mathrm{c}}, \sigma_{1}}(\mathbf{x})=\exp \left(-\left[\sigma_{1} \rho_{\mathbf{x}_{\mathrm{c}}}(\mathbf{x})\right]^{2}\right),
$$

where $\sigma_{1}>0$ and $\rho_{\mathbf{x}_{\mathbf{c}}}(\mathbf{x})=\cos ^{-1}\left(\mathbf{x} \cdot \mathbf{x}_{\mathbf{c}}\right)$ is the geodesic distance from $\mathbf{x}_{\mathbf{c}}$ to $\mathbf{x}$. For our numerical experiments, $\sigma_{1}=8$. Here the field $\mathbf{u}$ is infinitely smooth.

Example 2 To generate the second test case a non-smooth field $\mathbf{u}$ of the form (32), as done by Narcowich et al. [5], we replace $\boldsymbol{u}_{1, \mathbf{x}_{c}, \sigma}$ in (33) with $\mathrm{u}_{2, \mathbf{y}_{\mathrm{c}}, \sigma_{2}} \in \mathrm{H}^{3}(\mathrm{~S})$ (but $\mathrm{u}_{2, \mathrm{y}_{\mathrm{c}}, \sigma_{2}} \notin \mathrm{H}^{4}(\mathrm{~S})$ ) to be a $\mathrm{C}^{2}$-cubic spline that is positive for $\left|\sigma_{2} \rho_{\mathbf{y}_{\mathbf{c}}}(\mathbf{x})\right|<2$ and zero elsewhere on the unit sphere. That is the vector field $\mathbf{u}$ generated by

$$
\mathfrak{u}_{2, \mathbf{y}_{\mathbf{c}}, \sigma_{2}}(\mathbf{x})=\frac{\sigma_{2}^{3}}{12} \sum_{j=0}^{4}(-1)^{j}\left(\begin{array}{l}
4 \\
j
\end{array}\right)\left|\rho_{\mathbf{y}_{\mathbf{c}}}(\mathbf{x})-\frac{(j-2)}{\sigma_{2}}\right|^{3}
$$

satisfies $\mathbf{u} \in \mathrm{V} \cap \mathrm{H}^{\beta}$ (TS) with $\beta \in(2,3)$. For our numerical experiments, $\mathbf{y}_{\mathrm{c}}=\mathbf{p}(13 \pi / 18,13 \pi / 7)$ and $\sigma_{2}=4$.

We compute radial basis Galerkin approximations to both these fields in $V_{\tau, h_{N}}$ with $\tau=7 / 2,9 / 2$ and various values of $h_{N}$, generated using (17), (18), (21), (23) and $\mathrm{N}$ points on the sphere generated from an equal area partitioning algorithm by Saff and Kuijlaars [6]. Hence using Theorem 1 for $\mu=0,1$ we expect the estimated order of convergence (EOC) for Example 1 to be (at least) $2 \tau-\mu-1$ (because the field is infinitely smooth). For Example 2, Theorem 1 suggests EOC $\in(2-\mu, 3-\mu)$. For each $N$, the $L^{2}, H^{1}$-norm relative errors

$$
\left\|e_{\tau, h_{N}}\right\|=\left\|\mathbf{u}-\mathbf{u}_{\tau, h_{N}}\right\| /\|\mathbf{u}\|, \quad\left\|e_{\tau, h_{N}}\right\|_{H^{1}}=\left\|\mathbf{u}-\mathbf{u}_{\tau, h_{N}}\right\|_{H^{1}(\mathrm{TS})} /\|\mathbf{u}\|_{\mathrm{H}^{1}(\mathrm{TS})},
$$

are computed by approximating each $\mathrm{L}^{2}$-integral on the sphere by the Gaussrectangle cubature with 80,000 quadrature points on the unit sphere. $\mathrm{Nu}-$ merical results in Table 1 and Table 2 validate the efficiency of the radial basis Galerkin scheme for the smooth and non-smooth benchmark problems. 
TABLE 1: $\mathrm{L}^{2}(\mathrm{TS})$ and $\mathrm{H}^{1}(\mathrm{TS})$ errors and rates of convergence for Example 1.

\begin{tabular}{|c|c|c|c|c|c|c|}
\hline \multirow[t]{3}{*}{$\tau$} & $\mathrm{N}$ & 250 & 500 & 1000 & 2000 & 4000 \\
\hline & $h_{N}$ & 0.1610 & 0.1129 & 0.0794 & 0.0569 & 0.0396 \\
\hline & $\left\|e_{\tau, h_{N}}\right\|$ & $1.4 \mathrm{E}-1$ & $7.6 \mathrm{E}-2$ & $1.7 \mathrm{E}-2$ & $1.2 \mathrm{E}-3$ & $5.8 \mathrm{E}-5$ \\
\hline $7 / 2$ & EOC & & 1.77 & 4.14 & 7.81 & 8.58 \\
\hline & $\left\|e_{\tau, h_{N}}\right\|_{H^{1}}$ & $3.8 \mathrm{E}-1$ & $2.4 \mathrm{E}-1$ & $7.2 \mathrm{E}-2$ & $7.5 \mathrm{E}-3$ & $5.5 \mathrm{E}-4$ \\
\hline $7 / 2$ & EOC & & 1.34 & 3.42 & 6.75 & 7.20 \\
\hline & $\left\|e_{\tau, h_{N}}\right\|$ & $1.4 \mathrm{E}-1$ & $6.7 \mathrm{E}-2$ & $1.3 \mathrm{E}-2$ & $7.3 \mathrm{E}-4$ & $1.2 \mathrm{E}-5$ \\
\hline $9 / 2$ & EOC & & 2.14 & 4.62 & 8.63 & 11.43 \\
\hline $9 / 2$ & $\begin{array}{l}\left\|e_{\tau, h_{N}}\right\|_{H^{1}} \\
\text { EOC }\end{array}$ & $3.9 \mathrm{E}-1$ & $\begin{array}{l}2.1 \mathrm{E}-1 \\
1.71\end{array}$ & $\begin{array}{l}5.0 \mathrm{E}-2 \\
4.12\end{array}$ & $\begin{array}{l}3.8 \mathrm{E}-3 \\
7.73\end{array}$ & $\begin{array}{l}1.0 \mathrm{E}-4 \\
9.92\end{array}$ \\
\hline
\end{tabular}

In particular, results in Table 1 show that high order rate of convergence can be obtained using our method for smooth solutions. Further, the method can also achieve optimal order convergence even in the case of non-smooth Stokes flow, as demonstrated in Table 2. The standard EOC formula is close to the theoretical order of convergence only for sufficiently small mesh norm $h_{N}$. In our experiments since $h_{N}>10^{-2}$, we observe the oscillatory behaviour of the EOC values in Table 1 and Table 2.

\section{Conclusions}

We developed, analysed and demonstrated an efficient radial basis Galerkin method to simulate spherical surface Stokes equations. The main features of the meshless Galerkin algorithm include the advantage of simulating smooth Stokes flow surface velocity fields with high order accuracy and the ability to simulate non-smooth vector fields with optimal rate of convergence. Numerical experiments for benchmark smooth and non-smooth spherical surface vector fields validate the main features of the method and the associated theoretical analysis. 
TABLE 2: $\mathrm{L}^{2}(\mathrm{TS})$ and $\mathrm{H}^{1}(\mathrm{TS})$ errors and rates of convergence for Example 2.

\begin{tabular}{|c|c|c|c|c|c|c|}
\hline \multirow[t]{3}{*}{$\tau$} & $\mathrm{N}$ & 250 & 500 & 1000 & 2000 & 4000 \\
\hline & $h_{N}$ & 0.1610 & 0.1129 & 0.0794 & 0.0569 & 0.0396 \\
\hline & $\left\|e_{\tau, h_{N}}\right\|$ & $2.5 \mathrm{E}-2$ & $6.0 \mathrm{E}-3$ & $3.2 \mathrm{E}-3$ & $1.1 \mathrm{E}-3$ & $5.0 \mathrm{E}-4$ \\
\hline \multirow[t]{2}{*}{$7 / 2$} & EOC & & 4.06 & 1.73 & 3.02 & 2.35 \\
\hline & $\left\|e_{\tau, h_{N}}\right\|_{H^{1}}$ & $8.7 \mathrm{E}-2$ & $3.9 \mathrm{E}-2$ & $2.7 \mathrm{E}-2$ & $1.4 \mathrm{E}-2$ & $8.7 \mathrm{E}-3$ \\
\hline \multirow{2}{*}{$7 / 2$} & EOC & & 2.21 & 1.09 & 1.90 & 1.36 \\
\hline & $\| e_{\tau, h_{x}}$ & $2.3 \mathrm{E}-2$ & $5.7 \mathrm{E}-3$ & $2.9 \mathrm{E}-3$ & $1.1 \mathrm{E}-3$ & $4.6 \mathrm{E}-4$ \\
\hline \multirow[t]{2}{*}{$9 / 2$} & EOC & & 4.02 & 1.84 & 2.90 & 2.48 \\
\hline & $\left\|e_{\tau, h_{N}}\right\|_{H^{1}}$ & $7.9 \mathrm{E}-2$ & $3.9 \mathrm{E}-2$ & $2.5 \mathrm{E}-2$ & $1.3 \mathrm{E}-2$ & $8.2 \mathrm{E}-3$ \\
\hline $9 / 2$ & $\mathrm{EOC}$ & & 1.99 & 1.23 & 1.79 & 1.45 \\
\hline
\end{tabular}

Acknowledgements The support of the Australian Research Council is gratefully acknowledged.

\section{References}

[1] E. Fuselier, F. Narcowich, J. D. Ward, and G. Wright. Error and stability estimates for surface-divergence free RBF interpolants on the sphere. Math. Comp., 78:2157-2186, 2009. doi:10.1090/S0025-5718-09-02214-5 C64, C66

[2] M. Ganesh, Q. T. Le Gia, and I. H. Sloan. A pseudospectral quadrature method for Navier-Stokes equations on rotating spheres. Math. Comp., 80:1397-1430, 2011. doi:doi:10.1090/S0025-5718-2010-02440-8 C58, C60

[3] A. A. Il'in. The Navier-Stokes and Euler equations on two dimensional manifolds. Math. USSR. Sbornik, 69:559-579, 1991. doi:10.1070/SM1991v069n02ABEH002116 C59, C60, C61 
[4] F. J. Narcowich and J. D. Ward. Scattered data interpolation on spheres: error estimates and locally supported basis functions. SIAM J. Math. Anal., 33:1393-1410, 2002. doi:10.1137/S0036141001395054 C63

[5] F. J. Narcowich, J. D. Ward, and G. B. Wright. Divergence-free RBFs on surfaces. J. Fourier Anal. Appl., 13:643-663, 2007. doi:10.1007/s00041-006-6903-2 C59, C64, C67, C68

[6] E. B. Saff and A. B. J. Kuijlaars. Distributing many points on a sphere. Math. Intelligencer, 19:5-11, 1997. doi:10.1007/BF03024331 C65, C68

[7] H. Wendland. Piecewise polynomial, positive definite and compactly supported radial functions of minimal degree. Advances in Comp. Math., 4:389-396, 1995. doi:10.1007/BF02123482 C63

[8] D. L. Williamson, J. B. Drake, J. J. Hack, R. Jakob, and P. N. Swarztrauber. A standard test set for numerical approximations to the shallow water equations in spherical geometry. J. Comput. Phys., 102:211-224, 1992. doi:10.1016/0021-9991(92)90060-C C67

\section{Author addresses}

1. Mahadevan Ganesh, Department of Mathematical and Computer Sciences, Colorado School of Mines, Golden, CO 80401. USA. mailto:mganesh@mines . edu

2. Quoc Thong Le Gia, School of Mathematics and Statistics, University of New South Wales, Sydney, NSW 2052, Australia. mailto:qlegia@unsw.edu.au 